\title{
Nondegeneracy of Solution to the Allen-Cahn Equation with Regular Triangle Symmetry
}

\author{
Yong Liu, Jun Wang \\ Department of Mathematics and Physics, North China Electric Power University, Beijing, China \\ Email: liuyong@ncepu.edu.cn, junwang3000@163.com
}

Received 20 February 2014; revised 20 March 2014; accepted 28 March 2014

Copyright (C) 2014 by authors and Scientific Research Publishing Inc.

This work is licensed under the Creative Commons Attribution International License (CC BY). http://creativecommons.org/licenses/by/4.0/

(c) (i) Open Access

\section{Abstract}

The Allen-Cahn equation on the plane has a 6-end solution $U$ with regular triangle symmetry. The angle between consecutive nodal lines of $U$ is $\pi / 3$. We prove in this paper that $U$ is nondegenerated in the class of functions possessing regular triangle symmetry. As an application, we show the existence of a family of solutions close to $U$.

\section{Keywords}

\section{Allen-Cahn Equation, Multiple-End Solution, Nondegeneracy}

\section{Introduction}

We are interested in the following Allen-Cahn equation

$$
-\Delta u(x, y)=u(x, y)-u^{3}(x, y),|u|<1, z=(x, y) \in R^{2} .
$$

There have been a lot of work on this equation during the last two decays (see for example [1]-[5] and the references there in). An important class of solutions to (1.1) is the multiple-end solutions. By definition, a solution $u$ to (1.1) is called a multiple-end solution, if outside a large ball, the nodal curves of $u$ are asymptotic to finitely many half straight lines. One knows that these solutions have finite Morse index, and one also expects that any solution with finite Morse index should be a multiple-end solution. The most simple example of a multiple-end solution is $H\left(a_{1} x+a_{2} y+b\right)$, where $a_{1}^{2}+a_{2}^{2}=1, b \in R$, and $H$ is the heteroclinic solution: $H^{\prime \prime}+H-H^{3}=0, H(0)=0, H( \pm \infty)= \pm 1$. It is also well known ([6]) that for each $k \in N, k>1,(1.1)$ has a solution $U_{k}$ with regular polygon symmetry. More precisely, the nodal set of $U_{k}$ consists of $k$ straight lines

$$
y \cos (i \pi / k)=x \sin (i \pi / k), i=0, \cdots, k-1 \text {. }
$$


In the polar coordinate $(\rho, \theta)$ (we abuse the notation and use the same symbol for the function $U_{k}$ in the $(x, y)$ or $(\rho, \theta)$ coordinate):

$$
\begin{gathered}
U_{k}(\rho, \theta)=-U_{k}(\rho, \theta+\pi / k), \\
U_{k}(\rho, \theta)=U_{k}(\rho, \pi / k-\theta) .
\end{gathered}
$$

We could also assume $U_{k}(1, \pi / 2 k)<0$. In the special case $k=2$, the solution $U_{2}$ is called saddle solution. It turns out that $U_{2}$ is not isolated in the set of 4-end solutions. Actually, there is a family of 4-end solutions to (1.1) containing $U_{2}$ and the solutions $u_{\varepsilon}$ whose nodal lines are two almost parallel curves which are close to the solutions of the Toda system, see [7] [8]. In [9], it is shown that for each $k$, there is a family of $2 k$-end solutions whose nodal lines are close to suitable solutions of the classical Toda system. Intuitively, these solutions are in some sense on the boundary of the moduli space of $2 k$-end solutions and it is natural to expect that they are on the same connected component as $U_{k}$. In particular, one expects that around $U_{k}$, there should be a family of $2 k$-end solutions to (1.1). While this is true for $k=2$, in this paper, we will focus our attention on the solution $U_{3}$.

To state our result in a precise way, let us introduce some notations.

We will use $U$ to denote $U_{3}$. Let $L$ be the linearized operator around $U$ :

$$
L \phi=-\Delta \phi+\left(3 U^{2}-1\right) \phi .
$$

Our main theorem is the following nondegeneracy result:

Theorem 1.1 Assume $\phi$ is a $C^{2}$ solution of the equation $L \phi=0$ and

$$
\lim _{|z| \rightarrow \infty} \phi(z)=0 .
$$

Suppose furthermore that in the polar coordinate $(\rho, \theta)$,

$$
\begin{gathered}
\phi(\rho, \theta)=\phi(\rho, \pi-\theta), \\
\phi(\rho, \theta)=\phi(\rho, \theta+2 \pi / 3) .
\end{gathered}
$$

Then $\phi(z)=0$.

With the help of this theorem and the moduli space theory developed in [10], we have the following

Corollary 1.1 There is a family of 6-end solutions $U_{\varepsilon}$, different from $U$, such that $U_{\varepsilon} \rightarrow U$ in $C_{\text {loc }}^{2}\left(R^{2}\right)$ as $\varepsilon \rightarrow 0$ and in the polar coordinate,

$$
\begin{gathered}
U_{\varepsilon}(\rho, \theta)=U_{\varepsilon}(\rho, \pi-\theta), \\
U_{\varepsilon}(\rho, \theta)=U_{\varepsilon}(\rho, \theta+2 \pi / 3) .
\end{gathered}
$$

\section{Proof of Theorem 1.1}

To prove our main theorem, we will use the ideas developed in [11]. Assume to the contrary that $\phi$ is not identically zero. As a first step, we show that $\phi$ has the same symmetry as the function $U^{2}$.

Lemma 2.1 Under the assumption of Theorem 1.1,

$$
\phi(\rho, \theta)=\phi(\rho, \theta+\pi / 3) .
$$

Proof. The crucial observation is that since $U^{2}(\rho, \theta)=U^{2}(\rho, \theta+\pi / 3)$,

$$
-\Delta[\phi(\rho, \theta+\pi / 3)]+\left(3 U^{2}(\rho, \theta)-1\right) \phi(\rho, \theta+\pi / 3)=0 .
$$

Note that the Laplacian operator is taken with respect to the $(x, y)$ variable and in the Equation (2.1) the function is expressed in the polar coordinate.

Consider the function

$$
\Phi(\rho, \theta)=\phi(\rho, \theta)-\phi(\rho, \theta+\pi / 3) .
$$

It follows from (2.1) that $\Phi$ is also in the kernel of $L$, that is, 


$$
L \Phi=0
$$

It will then suffice to show $\Phi=0$.

By the definition of $\Phi$ and the symmetric of $\phi$, we have

$$
\begin{gathered}
\Phi(\rho, \pi / 3)=\phi(\rho, \pi / 3)-\phi(\rho, 2 \pi / 3)=0, \\
\Phi(\rho, 2 \pi / 3)=\phi(\rho, 2 \pi / 3)-\phi(\rho, \pi)=0 .
\end{gathered}
$$

On the other hand, the solution $U$ itself satisfies

$$
\begin{gathered}
U(\rho, \pi / 3)=0, \\
U(\rho, 2 \pi / 3)=0 .
\end{gathered}
$$

Moreover, in the region $\Omega=\{(\rho, \theta): \rho>0, \theta \in(\pi / 3,2 \pi / 3)\}, U$ is positive and is a supersolution of the operator $L$ :

$$
L U=-\Delta U+\left(3 U^{2}-1\right) U=2 U^{3}>0, \quad z \in \Omega .
$$

Denoting $h=U^{-1} \Phi$, then it is well defined in $\Omega$. From (2.2) and (2.3), we get

$$
\operatorname{div}\left(U^{2} \nabla h\right)=2 U^{3} \Phi .
$$

Following similar arguments as that of Lemma 2.1 in [11], with slight modification (one should take care of the fact that the right hand side of (2.4) is not identically zero), we could obtain $h \equiv c$ for some constant $c$, which implies that

$$
\Phi=c U .
$$

Since $\Phi$ decays to zero at infinity, we conclude $c=0$ and then $\Phi=0$. This completes the proof of this lemma.

Let $N=\left\{z \in R^{2}: \phi(z)=0\right\}$ be the nodal set of $\phi$. We proceed to show that $N$ is bounded.

Lemma 2.2 There exists a constant $C$ such that

$$
N \subset\left\{z \in R^{2}:|z| \leq C\right\} \text {. }
$$

Proof. We first show that for each connected component $M$ of $N$, there exists a constant $C_{M}$ such that $M$ is contained in the ball of radius $C_{M}$ :

$$
M \subset\left\{z \in R^{2}:|z| \leq C_{M}\right\} .
$$

We argue by contradiction and assume that $M$ is unbounded.

Case 1. $M \cap\{(0, y): y>0\} \neq \varnothing$.

In this case, we will consider $M^{\prime}=M \cap \Omega$. By the symmetry property of $\phi, M^{\prime}$ is still connected and unbounded. Therefore, one could find a continuous and piecewise smooth curve contained in $\bar{\Omega}$ :

$$
\eta: R \rightarrow M^{\prime}
$$

such that $|\eta( \pm s)| \rightarrow+\infty$, as $s \rightarrow+\infty$. One then could consider the nodal domain $D$ of $\phi$ contained in $\Omega$ whose boundary is the image $\eta(R)$ of $\eta$ (one could assume without loss of generality that $\eta$ does not have self intersection, otherwise, the presence of the supersolution $U$ yields a contradiction). Since $U$ is a positive supersolution of $L$ in $D$ similar arguments as Lemma 2.1 implies that $\Phi=c U$ in $D$ for some constant $c$, which is a contradiction.

Case 2. $M \cap\{(0, y): y>0\}=\varnothing$.

In this case, consider the region $\Lambda=\{\rho>0, \theta \in(\pi / 2,5 \pi / 6)\}$. Using symmetries of $\phi$, we could assume $M \subset \Lambda \cup\{(0,0)\}$. But in $\Lambda, U_{x}>0$ (This follows from a moving plane argument, see for example [12]) and $L U_{x}=0$, in particular, it is a positive supersolution. Hence similar arguments as in Lemma 2.1 imply that $\phi=0$, a contradiction. 
Hence each connected component $M$ is contained in a ball. To prove the assertion of this lemma, it will be suffice to show that there are only finite many connected components. We will assume to the contrary that there are infinite many of them. Then for each $r_{1}$ large, one could find a nodal domain of $\phi$ which is contained in

$$
\Sigma:=\left\{(\rho, \theta): \theta \in[0,2 \pi], r_{1}<\rho<r_{2}\right\},
$$

for some $r_{2}>r_{1}$. But when $r_{1}$ is large, since multiple-end solutions have finite Morse index, there is a positive supersolution $S$ in $\Sigma$ :

$$
L S>0, S>0 \text {, in } \Sigma \text {. }
$$

This contradicts with the maximum principle. The proof is thus completed. $\square$

Now we are ready to prove our main theorem.

Proof of Theorem 1. Suppose $\phi$ is not identically zero. By the previous lemmas, we could assume that $\phi(z)>0$ when $z$ is large enough, say for $|z|>r_{0}$.

Let us consider the projection of $\phi$ onto $H^{\prime}$. That is, we define the function

$$
g(x):=\int_{R} \phi(x, y) H^{\prime}(y) \mathrm{d} y, \quad x>r_{0} .
$$

Note that $H^{\prime}>0$ and hence $g(x)>0$.

We compute

$$
\begin{aligned}
g^{\prime \prime}(x) & =\int_{R} \partial_{x}^{2} \phi(x, y) H^{\prime}(y) \mathrm{d} y \\
& =\int_{R}\left[-\partial_{y}^{2} \phi+\left(3 U^{2}-1\right) \phi\right] H^{\prime}(y) \mathrm{d} y \\
& =\int_{R}\left[-H^{\prime \prime \prime}+\left(3 U^{2}-1\right) H^{\prime}\right] \phi \mathrm{d} y \\
& =3 \int_{R}\left(U^{2}-H^{2}\right) \phi H^{\prime} \mathrm{d} y
\end{aligned}
$$

Since $U^{2}-H^{2}<0$ (This could be seen from the construction of $U$ ), we find that $g^{\prime \prime}(x)<0$ for $x$ large. On the other hand, by the assumption, $\phi(z) \rightarrow 0$, as $|z| \rightarrow+\infty$. Therefore as $x$ goes to infinity, $g(x)$ goes to zero. This contradicts with the fact that $g^{\prime \prime}>0$ and the proof is thus completed.

A simple application of Theorem 1.1 is Corollary 1.1.

Proof of Corollary 1.1 By Theorem 1.1, the solution $U$ is nondegenerated in the class of functions having regular triangle symmetry. Then we could use the moduli space theory developed in [10], in suitable functional spaces having these symmetry. This theory tells us that locally around $U$ (in certain natural topology), the solution set has a structure of one dimensional real analytic manifold. Hence we get a family of 6-end solution $U_{\varepsilon}$ satisfying the property stated in Corollary 1.1 .

Remark 2.1 By the moduli space theory, the angles of consecutive ends of $U_{\varepsilon}$ will be close to $\pi / 3$. We conjecture that these angles should be exactly equal to $\pi / 3$. But this seems to be a difficult problem.

\section{Acknowledgements}

Y. Liu is partially supported by NSFC grant 11101141 and the Fundamental Research Funds for the Central Universities 13MS39.

\section{References}

[1] Ambrosio, L. and Cabre, X. (2000) Entire Solution of Semilinear Elliptic Equations in $R^{3}$ and a Conjecture of De Giorgi. Journal of the American Mathematical Society, 13, 725-739. http://dx.doi.org/10.1090/S0894-0347-00-00345-3

[2] Del Pino, M. Kowalczyk M. and Wei, J. (2011) On De Giorgi’s Conjecture in Dimension $N \geq 9$. Annals of Mathematics, 174, 1485-1569.

[3] Ghoussoub, N. and Gui, C. (1998) On a Conjecture of De Giorgi and Some Related Problems. Mathematische Annalen, 311, 481-491. http://dx.doi.org/10.1007/s002080050196

[4] Ghoussoub, N. and Gui, C. (1998) On a Conjecture of De Giorgi and Some Related Problems. Mathematische Annalen, 311, 121-132. http://dx.doi.org/10.1007/s002080050196

[5] Savin, O. (2010) Phase Transitions, Minimal Surfaces and a Conjecture of De Giorgi. Current Developments in Ma- 
thematics, 2009, 59-113.

[6] Alessio, F., Calamai, A. and Montecchiari, P. (2007) Saddle-Type Solutions for a Class of Semilinear Elliptic Equations. Advances in Differential Equations, 12, 361-380.

[7] Kowalczyk, M., Liu, Y. and Pacard, F. (2012) The Space of 4-Ended Solutions to the Allen-Cahn Equation in the Plane. Annales de l'Institut Henri Poincaré (C) Analyse Non Linéaire, 29,761-781. http://dx.doi.org/10.1016/j.anihpc.2012.04.003

[8] Kowalczyk, M., Liu, Y. and Pacard, F. (2013) The Classification of Four-End Solutions to the Allen-Cahn Equation on the Plane. Analysis and PDE, 6, 1675-1718.

[9] Del Pino, M., Kowalczyk, M., Pacard, F. and Wei, J. (2010) Multiple-End Solutions to the Allen-Cahn Equation in $R^{2}$. Journal of Functional Analysis, 258, 458-503. http://dx.doi.org/10.1016/j.jfa.2009.04.020

[10] Del Pino, M., Kowalczyk, M. and Pacard, F. (2013) Moduli Space Theory for the Allen-Cahn Equation in the Plane. Transactions of the American Mathematical Society, 365, 721-766. http://dx.doi.org/10.1090/S0002-9947-2012-05594-2

[11] Kowalczyk, M. and Liu, Y. (2011) Nondegeneracy of the Saddle Solution of the Allen-Cahn Equation on the Plane. Proceedings of the American Mathematical Society, 139, 4319-4329. http://dx.doi.org/10.1090/S0002-9939-2011-11217-6

[12] Gui, C. (2008) Hamiltonian Identities for Elliptic Partial Differential Equations. Journal of Functional Analysis, 254 904-933. http://dx.doi.org/10.1016/j.jfa.2007.10.015 